\title{
Characterization of Nanostructured Polymer Films
}

\section{RODNEY PRIESTLEY}

\section{TRUSTEES OF PRINCETON UNIVERSITY}

\section{$12 / 23 / 2014$}

Final Report 
Public reporting burden for this collection of information is estimated to average 1 hour per response, including the time for reviewing instructions, searching data sources,

gathering and maintaining the data needed, and completing and reviewing the collection of information. Send comments regarding this burden estimate or any other aspect of this collection of information, including suggestions for reducing this burden to Washington Headquarters Service, Directorate for Information Operations and Reports,

1215 Jefferson Davis Highway, Suite 1204, Arlington, VA 22202-4302, and to the Office of Management and Budget,

Paperwork Reduction Project (0704-0188) Washington, DC 20503.

PLEASE DO NOT RETURN YOUR FORM TO THE ABOVE ADDRESS.
1. REPORT DATE (DD-MM-YYYY)
2. REPORT DATE
TYPE: Final Report

TYPE: Final Report

4. TITLE AND SUBTITLE

Characterization of Nanostructured Polymer Films

5a. CONTRACT NUMBER

FA9550-12-1-0223

5b. GRANT NUMBER

5c. PROGRAM ELEMENT NUMBER

6. AUTHOR(S)

5d. PROJECT NUMBER

Priestley, Rodney D., Christie, Dane, Shepard, Kimberly B., Sosa,

Chris, Zhang, Chuan, Hyuncheol, Jeong

5e. TASK NUMBER

5f. WORK UNIT NUMBER

\section{PERFORMING ORGANIZATION NAME(S) AND ADDRESS(ES)}

The Trustees of Princeton Univeristy

8. PERFORMING ORGANIZATION

PO Box 36

Princeton, NJ 08544-0036

9. SPONSORING/MONITORING AGENCY NAME(S) AND ADDRESS(ES)

10. SPONSOR/MONITOR'S ACRONYM(S)

AF Office of Scientific Research

875 North Randolph Street, RM 3112

Arlington, VA 22203

11. SPONSORING/MONITORING AGENCY REPORT NUMBER

\section{DISTRIBUTION AVAILABILITY STATEMENT}

DISTRIBUTION A: Distribution approved for public release.

\section{SUPPLEMENTARY NOTES}

\section{ABSTRACT}

In 2011, we discovered that polymer films with exceptional thermal and kinetic stability could be formed by Matrix Assisted Pulsed Laser Evaporation (MAPLE) onto substrates held at low temperatures compared to the glass transition temperature $(\mathrm{Tg})$ of the polymer. The unique and unprecedented combination of properties is due to the film morphology, i.e., the films are nanostructured. The aim of this proposal was to understand the mechanism of film formation and to characterize the nanoscale building blocks of the stable glasses. Recently, we characterized the transport, i.e., time-of-flight, and nanoscale thermal properties of amorphous polymer nanoglobules fabricated via Matrix-Assisted Pulsed Laser Deposition (MAPLE). We discovered that stability in nanostrutured glasses was due to the nanoscale stability of the materials' building blocks, i.e., nanoglobules.

\section{SUBJECT TERMS}

Polymer Glasses, MAPLE, Glass Transition Temperature

\begin{tabular}{|l|l|l|l|l|}
\hline 16. SECURITY CLASSIFICATION OF: & 17. LIMITATION OF & 18. NUMBER \\
OF. REPORT & b. ABSTRACT & c. THIS PAGE & & \\
& & & & \\
\hline
\end{tabular}

19a. NAME OF RESPONSIBLE PERSON

19b. TELEPONE NUMBER (Include area code) 
1. REPORT DATE. Full publication date, including day, month, if available. Must cite at lest the year and be Year 2000 compliant, e.g., 30-06-1998; xx-08-1998; xx-xx-1998.

2. REPORT TYPE. State the type of report, such as final, technical, interim, memorandum, master's theis, progress, quarterly, research, special, group study, etc.

3. DATES COVERED. Indicate the time during which the work was performed and the report was written, e.g., Jun 1997 - Jun 1998; 1-10 Jun 1996; May - Nov 1998; Nov 1998.

4. TITLE. Enter title and subtitle with volume number and part number, if applicable. On classified documents, enter the title classification in parentheses.

5a. CONTRACT NUMBER. Enter all contract numbers as they appear in the report, e.g. F33615-86-C-5169.

5b. GRANT NUMBER. Enter all grant numbers as they apperar in the report, e.g. 1F665702D1257.

5c. PROGRAM ELEMENT NUMBER. Enter all program element numbers as they appear in the report, e.g. AFOSR-82-1234.

5d. PROJECT NUMBER. Enter al project numbers as they appear in the report, e.g. 1F665702D1257; ILIR.

5e. TASK NUMBER. Enter all task numbers as they appear in the report, e.g. 05; RF0330201; T4112.

5f. WORK UNIT NUMBER. Enter all work unit numbers as they appear in the repor, e.g. 001; AFAPL30480105.

6. AUTHOR(S). Enter name(s) of person(s) responsible for writing the report, performing the research, or credited with the content of the report. The form of entry is the last name, first name, middle initial, and additional qualifiers separated by commas, e.g. Smith, Richard, Jr.

7. PERFORMING ORGANIZATION NAME(S) AND ADDRESS(ES). Self-explanatory.
8. PERFORMING ORGANIZATION REPORT NUMBER.

Enter all unique alphanumeric report numbers assigned by the performing organization, e.g. BRL-1234; AFWL-TR-85-4017-Vol-21-PT-2.

\section{SPONSORING/MONITORS AGENCY NAME(S)}

AND ADDRESS(ES). Enter the name and address of the orgazination(s) financially responsible for and monitoring the work.

10. SPONSOR/MONITOR'S ACRONYM(S). Enter, if available, e.g. BRL, ARDEC, NADC.

11. SPONSOR/MONITOR'S REPORT NUMBER(S). Enter report number as assigned by the sponsoring/ monitoring agency, if available, e.g. BRL-TR-829; -215.

12. DISTRIBUTION/AVAILABILITY STATEMENT. Use agency-mandated availability statements to indicate the public availability or distribution limitations of the report. If additional limitations/restrictions or special markings are indicated, follow agency authorization procedures, e.g. RD/FRD, PROPIN, ITAR, etc. Include copyright information.

13. SUPPLEMENTARY NOTES. Enter information not included elsewhere such as: prepared in cooperation with; translation of; report supersedes; old edition number, etc.

14. ABSTRACT. A brief (approximately 200 words) factual summary of the most significant information.

15. SUBJECT TERMS. Key words or phrases identifying major concepts in the report.

16. SECURITY CLASSIFICATION. Enter security classification in accordance with security classification regulations, e.g. $\mathrm{U}, \mathrm{C}, \mathrm{S}$, etc. If this form contains classified information, stamp classification level on the top and bottom of this page.

17. LIMITATION OF ABSTRACT. This block must be completed to assign a distribution limitation to the abstract. Enter UU (Unclassified Unlimited) or SAR (Same as Report). An entry in this block is necessary if the abstract is to be limited. 


\author{
AFOSR YIP: Characterization of Nanostructured Polymer Films \\ Rodney D. Priestley, Chemical and Biological Engineering, Princeton University, Princeton, NJ 08544
}

\title{
Final Report
}

\section{Summary}

In 2011, we discovered that polymer films with exceptional thermal and kinetic stability could be formed by Matrix Assisted Pulsed Laser Evaporation (MAPLE) onto substrates held at low temperatures compared to the glass transition temperature $\left(T_{g}\right)$ of the polymer. The unique and unprecedented combination of properties is due to the film morphology, i.e., the films are nanostructured, as illustrated in Figure 1. We have spent the past few years trying to understand the growth mechanism of film formation and directly connecting the nanostructure to overall film properties. This final report focuses on the mechanism of nanostructured stable glass formation and the properties of the nanoscale building blocks. We will also discuss our recent results on using MAPLE to engineer the crystal structure in thin films.

Energy Landscape of Glass. The development of Matrix Assisted Pulsed Laser Evaporation (MAPLE) has enabled the gentle deposition of large macromolecules, where degradation can be avoided during laser processing. In the MAPLE method, a pulsed laser ablates a

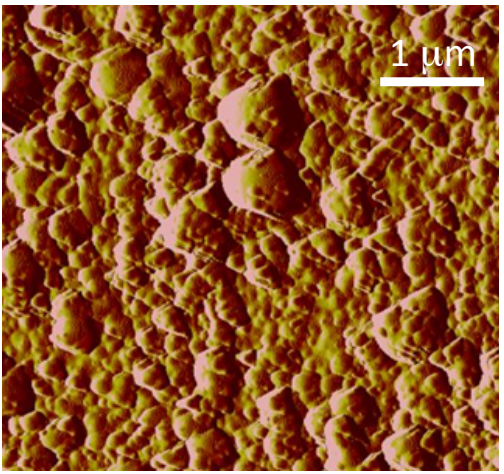

Figure 1. Surface topology of MAPLE-deposited PMMA film reveals the presence of surface nanoglobules. frozen target solution under vacuum, forming a plume, which is collected on a temperature-controlled substrate. As illustrated in Figure 1, we are able to generate, what we are calling, structured glasses. These glasses produced by MAPLE are significantly different from those formed by supercooling, i.e., the normal route to the glassy state. The differences in MAPLEdeposited glasses and those produced by supercooling are illustrated in Figure 2.

Surface Stability. We have suggested that the enhanced thermal stability of the nanostructured PMMA films is a direct result of the properties of the nanoglobular building blocks of film formation, i.e., the nanoglobules are thermally stable. We have shown that complete coalescence of surface nanoglobules occurs only at temperatures well above the normal $T_{g}$. In addition, we have indirectly investigated the thermal stability of the nanoglobules by examining the surface morphology via AFM of a series of PMMA films deposited at various substrate temperatures (see Figure 3 ). We note that the substrate temperature was the only processing parameter varied during these experiments, and AFM experiments were conducted at ambient conditions. The root-mean-square (RMS) roughness was observed to decrease from $\sim 140 \mathrm{~nm}$ at a low substrate temperature to $\sim 2 \mathrm{~nm}$ for a substrate temperature of $393 \mathrm{~K}$, as the surface nanostructure becomes less prominent. While the $T_{g}$ of the $5 \mathrm{~K}$ PMMA was $365 \mathrm{~K}$ (as determined by DSC on second heating at heating rate of $10 \mathrm{~K} / \mathrm{min}$ ), we

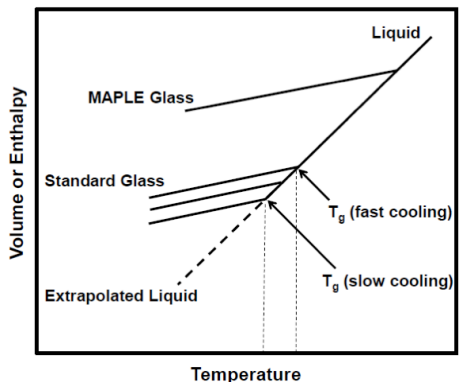

Figure 2. $\stackrel{\text { Temperature }}{\text { Schematic of }}$ volume or enthalpy vs. temperature comparing standard and MAPLEdeposited glasses. observed that surface nanoglobules were clearly present at $373 \mathrm{~K}$. The fact that surface globules were still present at $373 \mathrm{~K}$ is significant. At the $T_{g}$, the polymer segmental relaxation time is approximately $100 \mathrm{~s}$. The total deposition time of all experiments was $\sim 3600 \mathrm{~s}$. This indicates that sufficient time was allotted during the growth of the film for complete polymer chain relaxation, including relaxation of surface features. The presence of intact surface globules at a substrate temperature of $373 \mathrm{~K}$ suggests that the film has a $T_{g}$ greater than $373 \mathrm{~K}$, i.e., an enhanced $T_{g}$ compared to the normal value of $365 \mathrm{~K}$. Our results suggest that complete surface coalescence of the nanoglobules does not occur until the substrate temperature is at least $393 \mathrm{~K}$. We note that at $393 \mathrm{~K}$, the segmental relaxation time of the bulk polymer would be $\sim 0.1-1 \mathrm{~s}$. 


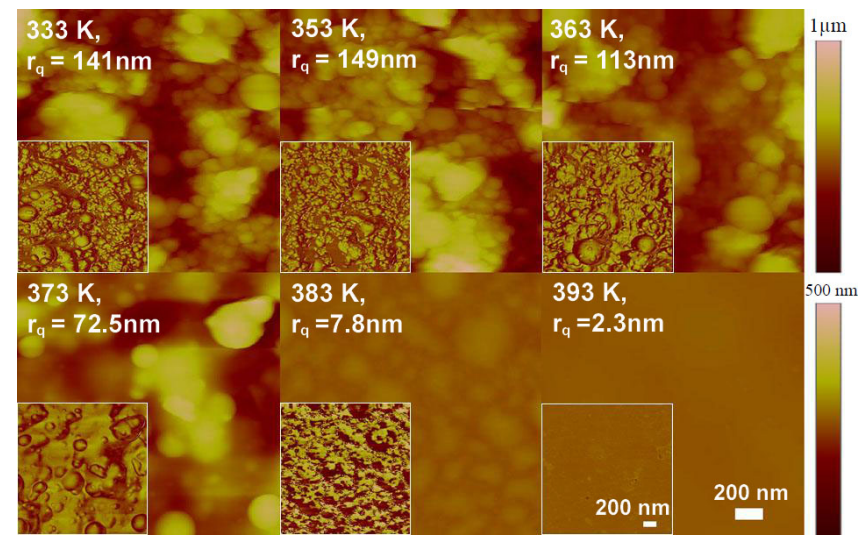

Figure 3. AFM height images and phase images (insets) of PMMA films deposited via MAPLE onto silica substrates held at various temperatures. The substrate temperature and RMS roughness for each film are given in the upper left corner of each panel. Nanoglobules persist on the surface at temperatures well above the bulk $T_{g}$ of $5 \mathrm{~K}$ PMMA. A substrate temperature of $393 \mathrm{~K}$ is required to fully eliminate surface nanoglobules.

Mechanism of MAPLE: Cooling Rate. We have argued that the significant enhancement in $T_{g}$ of MAPLE-deposited glasses is due to the cooling rate $(Q)$ experienced by the molecules during deposition. To quantitatively estimate the quench rate, we measured the fictive temperature $\left(T_{f}\right)$ as a function of cooling rate for PMMA and used the VFT expression to determine the cooling rate required to achieve the $T_{f}$ of MAPLE-deposited films. Data for the normalized fictive temperature (calculated by the Moynihan method) versus normalized cooling rate is shown on an Arrhenius plot in Figure 4. Cooling rate and fictive temperature are normalized by a standard cooling rate of $20 \mathrm{~K} / \mathrm{min}$, and the respective $T_{f}$ obtained at that cooling rate for each polymer. On the second set of axes in Figure 4, an Arrhenius plot of the VFT equation is superimposed. In the normalized form, the fictive temperatures of MAPLE-deposited glasses range from $T_{f, s} / T_{f}=$ $0.8-0.9$. These values correspond approximately to $\log \left(\mathrm{Q} / \mathrm{Q}_{\mathrm{S}}\right)=$ $7-10$, implying an effective cooling rate between $10^{8}-10^{11} \mathrm{~K} / \mathrm{min}$. The shaded region in Figure 4 indicates the range of $T_{f, s} / T_{f}$ values observed for MAPLE-deposited PMMA. Such high cooling rates

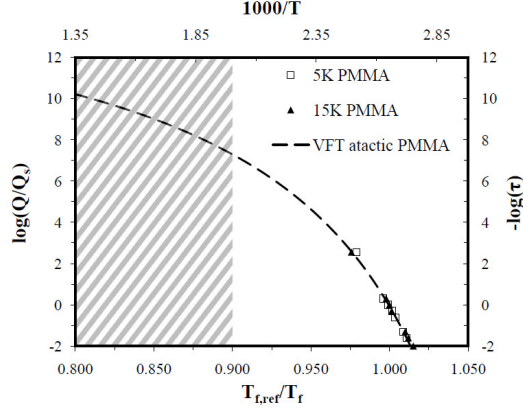

Figure 4: Arrhenius plots of normalized fictive temperature versus normalized cooling rate for $5 \mathrm{~K}$ and $15 \mathrm{~K}$ PMMA (left and bottom axes, symbols) and VFT fit for atactic PMMA (right and top axes, dotted line). would correspond to an increase in $T_{g}$ of $35-45 \mathrm{~K}$, which is in good agreement to what we measure for MAPLE-deposited glasses.

Mechanism of MAPLE: Nanostructure. We attribute the unique properties of MAPLE-deposited glasses to the nanostructure. The formation of a nanostructure in polymer films via MAPLE may occur during two distinct stages of the deposition process. In one case, the film may become nanostructured subsequent to material deposition by a mechanism such as evaporation-induced self-assembly, where directional evaporation of the solvent causes 2D ordering in the deposited film. In the second case, structure is set-in prior to the deposition of the desired material atop the substrate. That is, self-assembly of the nanoscale structures occurs within the time frame of the transfer process between the target and substrate. We are concerned with the latter mechanism of nanostructured film formation, as there is no evidence of solvent present in our films. In order to understand the origins of nanostructured films in the MAPLE process, we investigated the structure of sub-monolayer and monolayer films of PMMA deposited atop silicon oxide substrates. These experiments entailed characterizing, both visually and quantitatively, the nanostructure, i.e., nanoglobule morphology, of isolated nanoglobules and monolayer films as a function of deposition time. We also characterized the influence of polymer target concentration on the nanostructure morphology during the early stages of film growth. Our expectation was that by performing 
these measurements, new insight into the mechanism of the nanostructured film formation by MAPLE would be obtained.

To isolate the early-time MAPLE deposition behavior, we investigated the effect of MAPLE deposition time on the nanostructure of the deposited films. Five deposition times were examined $(2.5,5,10,20$ and $40 \mathrm{~min}$ ), while maintaining a constant polymer concentration of $0.1 \%$ by weight. AFM phase images for three of the resulting depositions $(2.5,10$ and $40 \mathrm{~min}$ ) are shown in Figure 5. The shortest deposition time (see Fig. 5(a)) yielded small, isolated nanoglobules distributed atop the silicon oxide substrate. The substrate was abraded with a razor to confirm the absence of an underlying planar layer. The individual nanoglobules resemble wetted droplets, whose geometry can be approximated as a spherical cap. The globules occupy approximately $16 \%$ of the substrate area, and the substrate is visible between the globules. After 10 min of MAPLE deposition, a near-continuous polymer film was formed, consisting of a monolayer of nanoglobules with minimal overlap (see Fig. 5(b)). In this deposition, a small number of globules were deposited on top of other globules, and the substrate was visible only in small discontinuities in the film. This 10 min deposition demonstrated a nanostructured polymer film with an average thickness of just $40 \mathrm{~nm}$. For a deposition time of $40 \mathrm{~min}$, the substrate was covered fully, and more overlapping of nanoglobules was observed (see Fig. 5(c)). To better quantify the differences in film nanostructure, we used image analysis software to measure the size distributions of the nanoglobules. We note that for depositions with times $\geq 5 \mathrm{~min}$, an ultrathin planar film 2-5 nm in thickness was present between the substrate and nanoglobules.

Figure $5 \mathrm{~d}$ is a histogram plot of the nanoglobule size distribution for the five different MAPLE deposition times. The size distributions for the two shortest deposition times, 2.5 and 5 min, were similar, with a narrow breadth (FWHM $=11$ and $13 \mathrm{~nm}$, respectively) and a peak at $20 \mathrm{~nm}$. The three longer deposition times (10, 20 and $40 \mathrm{~min}$ ) exhibited overlapping, broader size distributions with peaks at $25 \mathrm{~nm}$ $(\mathrm{FWHM}=22,23$ and $23 \mathrm{~nm}$, respectively). This implies two deposition regimes: an early-time regime, where more of the smaller globules are deposited and a second, slightly broader distribution, which stays constant, even at long deposition times. We suggest two potential mechanisms that may be responsible for the early time deposition regimes. First, the outer surface of the target, which is ablated during very early times, may not be identical in composition to the remainder of the target, due to a thin film of water vapor that freezes on the target prior to loading. Second, the gradual coalescence of deposited globules could be responsible for the initial increase in globule size with deposition time. The existence of two similar deposition regimes was also confirmed for a second target concentration $(0.5 \mathrm{wt} \%)$. The implication of the dual early-time deposition regimes is that it becomes possible to

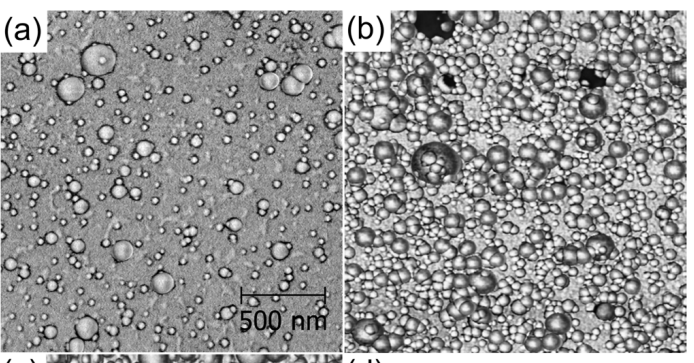

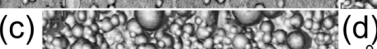

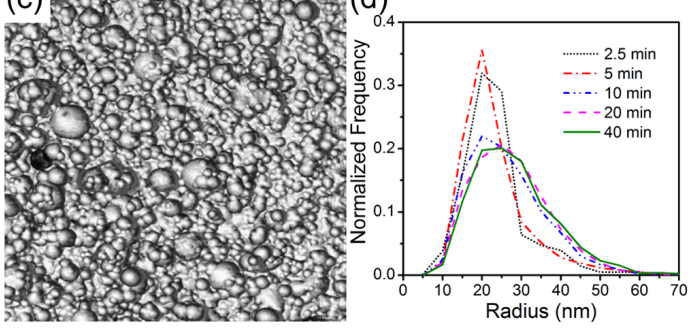

Figure 5. AFM phase images of PMMA films formed after (a) $2.5 \mathrm{~min}$, (b) $10 \mathrm{~min}$, and (c) $40 \mathrm{~min}$ of MAPLE deposition time. (d) Histogram plot of nanoglobule radius for five deposition times. establish a rule of thumb that the globule size distributions resulting from all depositions longer than the critical time are comparable to each other. This is useful when varying other MAPLE deposition parameters, such as target concentration. 
Earlier work, we briefly addressed the formation of MAPLE-deposited polymer nanoglobules within the context of the Zhigilei model of target ablation in the MAPLE process. Molecular dynamics simulations show that phase explosion causes polymer molecules to be ejected from the MAPLE target within clusters of solvent. In the simulation work, the size distribution of ejected clusters was characterized as a function of fluence and polymer target concentration, where the size was measured in the number of mers of polymer $(\mathrm{N}=100$ mers per chain) plus molecules of solvent in each cluster. A power law dependence of $\mathrm{N}^{-1.6}$ was identified. In Fig. 6(a), the size distribution data for films deposited from 0.05 and 0.1 $\mathrm{wt} \%$ solutions is plotted with $95 \%$ confidence intervals. The best power law fit was found for
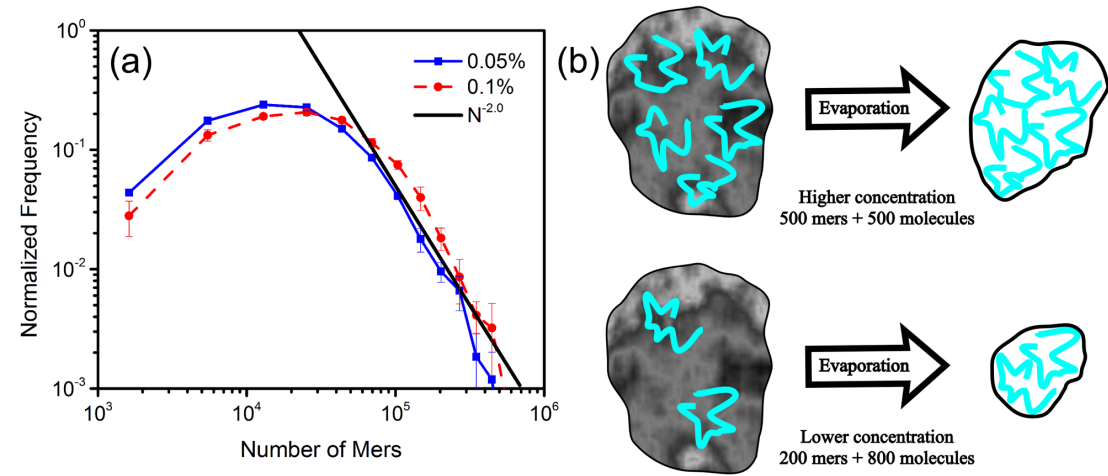

Figure 6: (a) Histogram plot of number of mers per nanoglobule for $0.05 \%$ (dotted red curve) and $0.1 \%$ (solid blue curve) MAPLEdeposited films, compared with $\mathrm{N}^{-2.0}$ scaling. (b) Schematic of nanoglobule formation from MAPLE-ejected polymer/solvent clusters. Equal sized clusters with different polymer concentrations will form differently sized nanoglobules after evaporation. both 0.05 and $0.1 \mathrm{wt} \%\left(\mathrm{~N}^{-2.1}\right.$ and $\mathrm{N}^{-1.9}$, respectively) over the radius range of $30 \mathrm{~nm} \leq \mathrm{R} \leq 65 \mathrm{~nm}$. An intermediate power law fit with exponent of $\mathrm{N}^{-2.0}$ was included in the graph in Fig. 6(a). For globules larger than $65 \mathrm{~nm}$, the number of globules present in each film is inadequate for our analysis to generate meaningful statistics, and thus these were not included in the fit. For globules smaller than $30 \mathrm{~nm}\left(<5 \times 10^{5}\right.$ mers $)$, the data no longer follow a power law expression. An outcome of this study is an explanation for the influence of concentration on the sizes polymer nanoglobules as depicted schematically in Fig. 6(b).

Properties of Isolated Nanoglobules. In earlier work, (Shepard, APA, 2013) we provided an indirect measurement of nanoglobule stability. In 2013, our focus has been on establishing a protocol to directly measure the thermal stability of isolated nanoglobules prepared via MAPLE. To achieve this, we performed short time MAPLE deposition to generate isolated nanoglobules. We subsequently, used AFM to measure the volume of nanoglobules as a function of temperature. Figure 7 illustrates data from such experiments (ACS Macro Letters 2014). Figure 7a compares the plots of normalized volume (normalized at room temperature) versus temperature for nanodroplets (prepared by annealing nanoparticles) and MAPLE nanoglobules. There is a notable difference in the volume response. Nanoglobules prepared by MAPLE have a pronounced drop in volume at high temperatures while those prepared from nanoparticles do not exhibit such behavior. Figures $7 \mathrm{~b}$ and $\mathrm{c}$ depict profiles of nanoglobules prepared by annealing a nanoparticle and MAPLE, respectively. Cleary, there is a dramatic volume shrinkage at a critical temperature of the MAPLE prepared nanoglobule. I would like to make two important points: 1) The temperature at which the volume reduction occurs is $\sim 20 \mathrm{~K}$ higher than the bulk glass transition temperature, and 2) The volume reduction is $\sim 30 \%$ of the film volume. Both of these metrics are consistent with our bulk measurements of MAPLE-deposited films. We hope to expand to nanoscale calorimetry in the future. 
We have build a time of flight measurement system to measure the velocity of isolated polymer nanoglobules during deposition. The time of flight (TOF) measurement system is designed to experimentally measure the time it takes laserablated material to travel from the target to the substrate. From this measurement, we can characterize the velocity and kinetic energy of the ablated material, to be used in an energy balance of the material deposition process. Inside the MAPLE chamber, we have installed a $6 \mathrm{MHz}$ quartz crystal microbalance system, positioned directly in front of the ablation plume. The crystal continually oscillates, and the frequency of the oscillation decreases as material is deposited onto the crystal's surface. Using an oscilloscope, we can take a "snapshot" in time of the crystal's oscillatory signal. We can synchronize the beginning of the snapshot to the time when a laser pulse strikes the target, using a photodetector positioned just outside the beam path. The snapshot stored in the oscilloscope is then analyzed using a fast Fourier transform algorithm in MATLAB to pinpoint the time at which the crystal's frequency changed, and hence, what time the material arrived at the crystal sensor. The velocity of MAPLE-deposited material has been investigated only in simulations, where it is reported on the order of $100-1000 \mathrm{~m} / \mathrm{s}$. Based on these calculations, we expect the timescale of our measurements will be tens to hundreds of microseconds, which is within the capabilities of the system. I report that the system is working and that we are able to measure velocity of MAPLEdeposited nanoglobules.

Macromolecular Deposition of Semi-Crystalline Polymers via MAPLE. We have very recently begun the gas-phase deposition of semi-crystalline polymers via MAPLE. Figure 5 displays Atomic Force Microscopy (AFM) images of a model crystallizable polymer, polyethylene oxide (PEO) deposited via MAPLE onto native silicon substrates
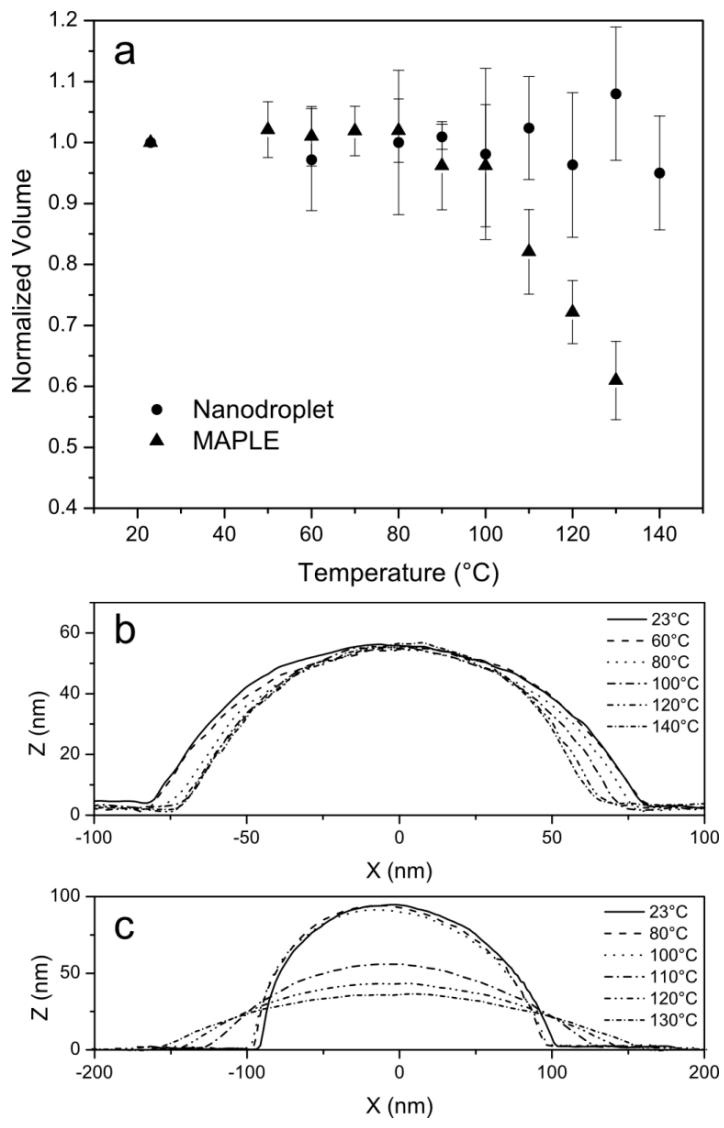

Figure 7: (a) Normalized volume of polymer nanodroplets and MAPLE-deposited nanoglobules as a function of temperature for large features $\left(V>10^{-21} \mathrm{~m}^{3}\right)$. (b) Profiles of a large nanodroplet's $X Z$ cross-section as it is heated. (c) Profiles of a large MAPLEdeposited nanoglobule's $X Z$ cross-section as it is heated. held at room temperature. Panels $\mathrm{A}, \mathrm{B}$, and $\mathrm{C}$ illustrate height images of a film deposited at $\sim 0.0001 \mathrm{~nm} / \mathrm{sec}$ for 120,240 , and $480 \mathrm{~min}$, respectively. While panel $D$ is the phase image of panel $C$. The images demonstrate that the size of the monolamellae increases with increasing deposition time. This phenomenon is a result of the films growth mechanism via the gas phase deposition process and illustrates a new means for engineering the morphology in semi-crystalline polymer thin films similar to that of growing crystalline films of small molecules via vapor deposition. 


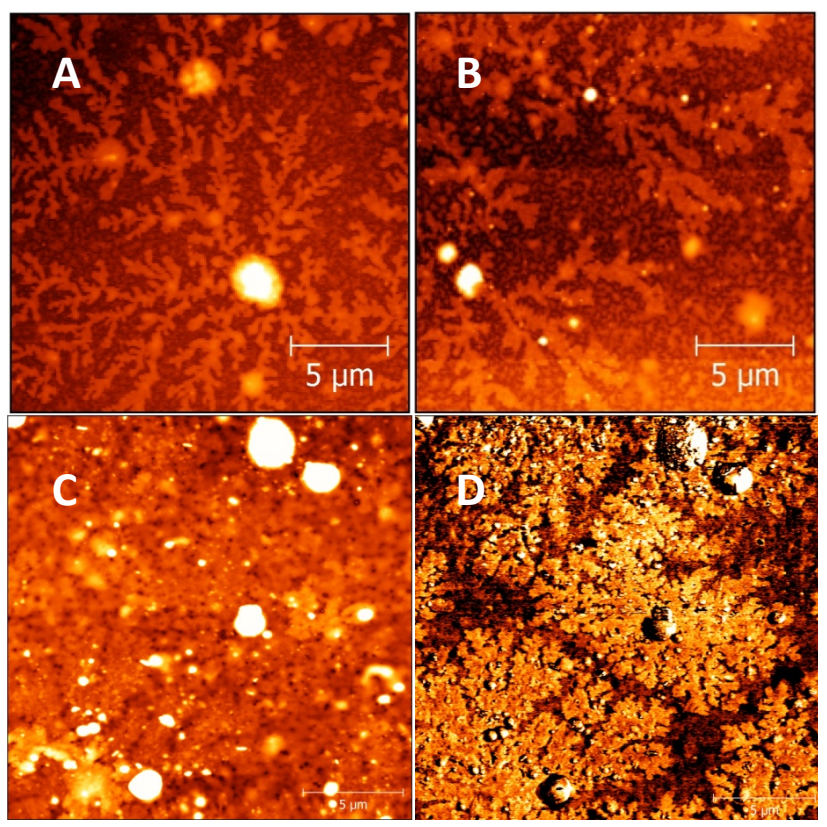

Figure 5: Development of dendritic arms of PEO on Si wafers with surface coverage after (A) 120 min (B) 240 min (C)-(D) 480 min deposition times. Panels A-C are height images while panel $D$ is a phase image. 


\section{Report Type}

Final Report

\section{Primary Contact E-mail}

Contact email if there is a problem with the report.

rpriestl@princeton.edu

\section{Primary Contact Phone Number}

Con tact phone number if there is a problem with the report

609-258-5721

\section{Organization / Institution name}

Princeton University

\section{Grant/Contract Title}

The full title of the funded effort.

(YIP 12) Characterization of Nanostructured Polymer Films

\section{Grant/Contract Number}

AFOSR assigned control number. It must begin with "FA9550" or "F49620" or "FA2386".

FA9550-12-1-0223

\section{Principal Investigator Name}

The full name of the principal investigator on the grant or con tract.

Rodney Dewayne Priestley

Program Manager

The AFOSR Program Manager currently assigned to the award

Joycelyn Harrison

Reporting Period Start Date

$07 / 01 / 2012$

\section{Reporting Period End Date}

$$
12 / 31 / 2014
$$

\section{Abstract}

In 2011, we discovered that polymer films with exceptional thermal and kinetic stability could be formed by Matrix Assisted Pulsed Laser Evaporation (MAPLE) onto substrates held at low temperatures compared to the glass transition temperature $(\mathrm{Tg})$ of the polymer. The unique and unprecedented combination of properties is due to the film morphology, i.e., the films are nanostructured. The aim of this proposal was to understand the mechanism of film formation and to characterize the nanoscale building blocks of the stable glasses. Recently, we characterized the transport, i.e., time- of-flight, and nanoscale thermal properties of am orphous polymer nanoglobules fabricated via Matrix-Assisted Pulsed Laser Deposition (MAPLE). We reported the first experimental measurement of the velocity of polymer during MAPLE processing and its connection to nanostructured film formation. A nanoscale dilatometry technique using atomic force microscopy was employed to directly measure the thermal properties of MAPLE-deposited polymer nanoglobules. Similarly to bulk stable polymer glasses deposited by MAPLE, polymer nanoglobules were found to exhibit enhanced thermal stability and low density despite containing only thousands of molecules. By directly connecting the exceptional properties of the nanostructured building blocks to DISTRIBUTION A: Distribution approved for public release. 
those of bulk stable glasses, we gained insight into the physics of glassy polymeric materials formed via vapor-assisted techniques.

Distribution Statement

This is block 12 on the SF298 form.

Distribution A - Approved for Public Release

Explanation for Distribution Statement

If th is is not approved for public release, please provide a short explanation. E.g., con tains proprietary information.

SF298 Form

Please attach your SF298 form. A blank SF298 can be found here. Please do not password protect or secure the PDF The maximum file size for an SF298 is 50MB.

ONRSF298.pdf

Upload the Report Document. File must be a PDF. Please do not password protect or secure the PDF . The maximum file size for the Report Document is 50MB.

AFOSR YIP Final Report .pdf

Upload a Report Document, if any. The maximum file size for the Report Document is $50 \mathrm{MB}$.

Archival Publications (published) during reporting period:

K. Shepard, D. Christie, C.L. Sosa, C.B. Arnold, R.D. Priestley*, Patchy Janus Particles with Tunable Roughness and Composition via Vapor-Assisted Deposition of Macromolecules, submitted.

K.B. Shepard, C.B. Arnold, R.D. Priestley*, Transport and Stability of Laser-Deposited Amorphous Polymer Nanoglobules, ACS Macro Letters, 3, 1046 (2014)

K. Shepard, C.B. Arnold, R.D. Priestley*, Origins of Nanostructure in Amorphous Polymer Coatings via Matrix Assisted Pulsed Laser Evaporation, Applied Physics Letters, 103, 123105 (2013)

K. Shepard, R.D. Priestley*, MAPLE Deposition of Macromolecules, Macromolecular Chemistry and Physics, 214, 862 (2013)

K. Shepard, Y. Guo, C.B. Arnold, R.D. Priestley`, Nanostructured Morphology of Polymer Films Prepared by Matrix Assisted Pulsed Laser Evaporation, Applied Physics A, 110, 771

Changes in research objectives (if any):

None

Change in AFOSR Program Manager, if any:

None

Extensions granted or milestones slipped, if any:

The proposal was converted into PECASE Award

\section{AFOSR LRIR Number}

LRIR Title

Reporting Period

Laboratory Task Manager

Program Officer

Research Objectives

Technical Summary

Funding Summary by Cost Category (by FY, \$K)

DISTRIBUTION A: Distribution approved for public release. 


\begin{tabular}{|l|l|l|l|} 
& Starting FY & FY+1 & FY+2 \\
\hline Salary & & & \\
\hline Equipment/Facilities & & & \\
\hline Supplies & & & \\
\hline Total & & & \\
\hline
\end{tabular}

\section{Report Document}

Report Document - Text Analysis

Report Document - Text Analysis

Appendix Documents

2. Thank You

E-mail user

Dec 16, 2014 21:48:13 Success: Email Sent to: rpriestl@princeton.edu 